COMMUNICATIONS IN

ANALYSIS AND GEOMETRY

Volume 14, Number 5, 883-905, 2006

\title{
A note on Perelman's LYH-type inequality
}

\author{
LEI NI
}

\begin{abstract}
We give a proof to the Li-Yau-Hamilton-type inequality claimed by Perelman on the fundamental solution to the conjugate heat equation. The rest of the paper is devoted to improving the known differential inequalities of $\mathrm{Li}-\mathrm{Yau}-\mathrm{Hamilton}$ type via monotonicity formulae.
\end{abstract}

\section{Introduction}

In [1], Perelman stated a Li-Yau-Hamilton type (also called differential Harnack) inequality for the fundamental solution of the conjugate heat equation on a manifold evolving by the Ricci flow. More precisely, let $\left(M, g_{i j}(t)\right)$ be a solution to Ricci flow:

$$
\frac{\partial}{\partial t} g_{i j}=-2 R_{i j}
$$

on $M \times[0, T]$ and let $H(x, y, \tau)=\mathrm{e}^{-f} /(4 \pi \tau)^{n / 2}$ (where $\left.\tau=T-t\right)$ be the fundamental solution to the conjugate heat equation $u_{\tau}-\Delta u+R u=0$. (More precisely we should write the fundamental solution as $H(y, t ; x, T)$, which satisfies $\left(-\partial / \partial t+\Delta_{y}+R(y, t)\right) H(y, t ; x, T)=0$ for any $(y, t)$ with $t<T$ and $\lim _{t \rightarrow T} \int_{M} H(y, t ; x, T) f(y, t) d \mu_{t}(y)=f(x, T)$.) Define

$$
v_{H}=\left[\tau\left(2 \Delta f-|\nabla f|^{2}+R\right)+f-n\right] H .
$$

Here, all the differentiations are taken with respect to $y$, and $n=\operatorname{dim}_{\mathbb{R}}(M)$. Then, $v_{H} \leq 0$ on $M \times[0, T]$. This result is a differential inequality of Li-Yau type [2], which has important applications in the later part of [1]. For example, it is essential in proving the pseudo-locality theorem in Section 10 of [1]. It is also crucial in localizing the entropy formula [3].

In Section 9 of [1], the following important differential equation

$$
\left(\frac{\partial}{\partial \tau}-\Delta+R\right) v_{u}=-2 \tau\left|R_{i j}+\nabla_{i} \nabla_{j} f-\frac{1}{2 \tau} g_{i j}\right|^{2} u
$$


is stated for any positive solution $u$ to the conjugate heat equation, whose integration on $M$ gives the celebrated entropy formula for the Ricci flow. One can consult various sources (e.g. [4]) for the detailed computations of this equation, which can also be done through a straightforward calculation, after knowing the result. Perelman [1] then proceeds with the proof of the claim $v_{H} \leq 0$ in a clever way by checking that for any $\tau_{*}$ with $T \geq \tau_{*}>0, \int_{M} v_{H}(y) h(y) d \mu_{\tau_{*}}(y) \leq 0$, for any smooth function $h(y) \geq 0$ with compact support. In order to achieve this, in [1] the heat equation $(\partial / \partial t-\Delta) h(y, t)=0$ with the "initial data" $h\left(y, T-\tau_{*}\right)=h(y)$ (more precisely $\left.t=T-\tau_{*}\right)$, the given compactly supported non-negative function, is solved. Applying (1.2) to $u(y, \tau)=H(x, y, \tau)$, one can easily derive as in [1], via integration by parts, that

$$
\frac{d}{d \tau} \int_{M} v_{H} h d \mu_{\tau}=-2 \int_{M} \tau\left|R_{i j}+\nabla_{i} \nabla_{j} f-\frac{1}{2 \tau} g_{i j}\right|^{2} H h d \mu_{\tau} \leq 0 .
$$

The Li-Yau-type inequality $v_{H} \leq 0$ then follows from the above monotonicity, provided the claim that

$$
\lim _{\tau \rightarrow 0} \int_{M} v_{H} h d \mu_{\tau} \leq 0
$$

The main purpose of this note is to prove (1.4) and hence provide a complete proof of the claim $v_{H} \leq 0$. This will be done in Section 3 after some preparations in Section 2. It was written in [1] that "it is easy to see" that $\lim _{\tau \rightarrow 0} \int_{M} v_{H} h d \mu_{\tau}=0$. It turns out that the proof found here needs to use some gradient estimates for positive solutions, quite precise estimate on the "reduced distance", a tool also introduced by Perelman in [1], and the monotonicity formula (1.3). (We shall focus on the proof of (1.4) for the case when $M$ is compact and leave the more technical details of generalizing it to the non-compact setting to the later refinements.) Indeed the claim that $\lim _{\tau \rightarrow 0} \int_{M} v_{H} h d \mu_{\tau}=0$ follows from a blow-up argument of [1], after we have established (1.4). Since our argument is a bit involved, this may not be the proof.

In Section 4, we derive several monotonicity formulae, which improve various $\mathrm{Li}-\mathrm{Yau}-\mathrm{Hamilton}$ inequalities for linear heat equation (systems) as well as for Ricci flow, including the original Li-Yau's inequality. In Section 5 , we illustrate their localization by applying a general scheme of [5]. 


\section{Estimates and results needed}

We shall collect some known results and derive some estimates needed for proving (1.4) in this section. We need the asymptotic behavior of the fundamental solution to the conjugate heat equation for small $\tau$. Let $d_{\tau}(x, y)$ be the distance function with respect to the metric $g(\tau)$. Let $B_{\tau}(x, r)\left(\operatorname{Vol}_{\tau}\right)$ be the ball of radius $r$ centered at $x$ (the volume) with respect to the metric $g(\tau)$.

Theorem 2.1. Let $H(x, y, \tau)$ be the fundamental solution to the (backward in $t)$ conjugate heat equation. Then, as $\tau \rightarrow 0$, we have that

$$
H(x, y, \tau) \sim \frac{\exp \left(-d_{0}^{2}(x, y) / 4 \tau\right)}{(4 \pi \tau)^{n / 2}} \sum_{j=0}^{\infty} \tau^{j} u_{j}(x, y, \tau)
$$

By (2.1), we mean that there exists $T>0$ and sequence $u_{j} \in C^{\infty}(M \times M \times$ $[0, T])$ such that

$$
H(x, y, \tau)-\frac{\exp \left(-d_{0}^{2}(x, y) / 4 \tau\right)}{(4 \pi \tau)^{n / 2}} \sum_{j=0}^{k} \tau^{j} u_{j}(x, y, \tau)=w_{k}(x, y, \tau)
$$

with

$$
w_{k}(x, y, \tau)=O\left(\tau^{k+1-n / 2}\right)
$$

as $\tau \rightarrow 0$, uniformly for all $x, y \in M$. The function $u_{0}(x, y, \tau)$ can be chosen so that $u_{0}(x, x, 0)=1$.

This result was proved in detail, for example in [6], when there is no zero order term $R(y, \tau) u(y, \tau)$ in the equation

$$
\frac{\partial}{\partial \tau} u-\Delta u+R u=0
$$

and replacing $d_{0}(x, y)$ by $d_{\tau}(x, y)$. However, one can check that the argument carries over to this case if one assumes that the metric $g(\tau)$ is $C^{\infty}$ near $\tau=0$. One can consult $[7,8]$ for intrinsic presentations.

Let

$$
\mathcal{W}_{h}(g, H, \tau)=\int_{M} v_{H} h d \mu_{\tau}
$$

where $h$ is the previously described solution to the heat equation. It is clear that for any $\tau$ with $T \geq \tau>0, \mathcal{W}_{h}(g, H, \tau)$ is a well-defined quantity. 
A priori it may blow up as $\tau \rightarrow 0$. It turns out that in our course of proving that $\lim _{\tau \rightarrow 0} \mathcal{W}_{h}(g, H, \tau) \leq 0$, we need to show first that there exists $C>0$, which may depend on the geometry of the Ricci flow solution $(M, g(\tau))$ defined on $M \times[0, T]$, but independent of $\tau($ as $\tau \rightarrow 0)$ so that $\mathcal{W}_{h}(g, H, \tau) \leq$ $C$ for all $T \geq \tau>0$. The following lemma (see also [5] for a localized version of it) supplies the key estimates for this purpose.

Lemma 2.2. Let $(M, g(t))$ be a smooth solution to the Ricci flow on $M \times$ $[0, T]$. Assume that there exist $k_{1} \geq 0$ and $k_{2} \geq 0$, such that the Ricci curvature $R_{i j}(g(\tau)) \geq-k_{1} g_{i j}(\tau)$ and $\max \left(R(y, \tau),|\nabla R|^{2}(y, \tau)\right) \leq k_{2}$, on $M \times[0, t]$.

(i) If $u \leq A$ is a positive solution to the conjugate heat equation on $M \times[0, T]$, then there exist $C_{1}$ and $C_{2}$ depending on $k_{1}, k_{2}$ and $n$ such that for $0<\tau \leq \min \left(1, T, 1 / 2 k_{2}\right)$,

$$
\tau \frac{|\nabla u|^{2}}{u^{2}} \leq\left(1+C_{1} \tau\right)\left(\log \left(\frac{A}{u}\right)+C_{2} \tau\right)
$$

(ii) If $u$ is a positive solution to the conjugate heat equation on $M \times$ $[0, T]$, then there exists $B$ depending on $(M, g(\tau))$ so that for $0 \leq \tau \leq$ $\min \left(T, 1 / 2 k_{2}, 1\right)$,

$$
\tau \frac{|\nabla u|^{2}}{u^{2}} \leq\left(2+C_{1} \tau\right)\left(\log \left(\frac{B}{u \tau^{n / 2}} \int_{M} u d \mu_{\tau}\right)+C_{2} \tau\right) .
$$

Remark 2.3. Here and thereafter we use the same $C_{i}(B)$ at different lines if they differ only by a constant depending on $n$. Notice that $\int_{M} u d \mu_{\tau}$ is independent of $\tau$ and equal to 1 if $u$ is the fundamental solution. The proof of the lemma given below is a modification of some arguments in [9].

Proof. Direct computation, using a unitary frame, gives

$$
\begin{aligned}
\left(\frac{\partial}{\partial \tau}-\Delta\right)\left(\frac{|\nabla u|^{2}}{u}\right)= & -\frac{2}{u}\left|u_{i j}-\frac{u_{i} u_{j}}{u}\right|^{2}+\frac{|\nabla u|^{2}}{u} R \\
& +\frac{-4 R_{i j} u_{i} u_{j}-2\langle\nabla(R u), \nabla u\rangle}{u} \\
\leq & (4+n) k_{1} \frac{|\nabla u|^{2}}{u}+2|\nabla R||\nabla u| \\
\leq & {\left[(4+n) k_{1}+1\right] \frac{|\nabla u|^{2}}{u}+k_{2} u }
\end{aligned}
$$


and

$$
\begin{aligned}
\left(\frac{\partial}{\partial \tau}-\Delta\right)\left(u \log \left(\frac{A}{u}\right)\right) & =\frac{|\nabla u|^{2}}{u}+R u-R u \log \left(\frac{A}{u}\right) \\
& \geq \frac{|\nabla u|^{2}}{u}-n k_{1} u-k_{2} u \log \left(\frac{A}{u}\right)
\end{aligned}
$$

Combining the above two equations, we have that

$$
\left(\frac{\partial}{\partial \tau}-\Delta\right) \Phi \leq 0
$$

where

$$
\Phi=\varphi \frac{|\nabla u|^{2}}{u}-\mathrm{e}^{k_{2} \tau} u \log \left(\frac{A}{u}\right)-2\left(k_{2}+n k_{1} \mathrm{e}^{k_{2}}\right) \tau u
$$

with

$$
\varphi=\frac{\tau}{1+\left[(4+n) k_{1}+1\right] \tau}
$$

which satisfies

$$
\frac{d}{d \tau} \varphi+\left[(4+n) k_{1}+1\right] \varphi<1 .
$$

By the maximum principle, we have that

$$
\varphi \frac{|\nabla u|^{2}}{u} \leq \mathrm{e}^{k_{2} \tau} u \log \left(\frac{A}{u}\right)+2\left(k_{2}+n k_{1} \mathrm{e}^{k_{2}}\right) \tau u .
$$

From this, one can derive (2.2) easily.

To prove the second part, we claim that for $u$, a positive solution to the conjugate heat equation, there exists a $C$ depending on $(M, g(\tau))$ such that

$$
u(y, \tau) \leq \frac{C}{\tau^{n / 2}} \int_{M} u(z, \tau) d \mu_{\tau}(z)
$$

This is a mean-value-type inequality, which can be proved via, for example, the Moser iteration. Here, we follow [9]. We may assume that $\sup _{y \in M, 0 \leq \tau \leq 1}$ $\tau^{n / 2} u(y, \tau)$ is finite. Otherwise, we may replace $\tau$ by $\tau_{\epsilon}=\tau-\epsilon$ and let $\epsilon \rightarrow 0$ after establishing the claim for $\tau_{\epsilon}$. Now let $\left(x_{0}, \tau_{0}\right) \in M \times[0,1]$ be such a 
space-time point that $\max \tau^{n / 2} u(y, \tau)=\tau_{0}^{n / 2} u\left(y_{0}, \tau_{0}\right)$. Then, we have that

$$
\sup _{M \times\left[\tau_{0} / 2, \tau_{0}\right]} u(y, t) \leq\left(\frac{2}{\tau_{0}}\right)^{n / 2} \tau_{0}^{n / 2} u\left(y_{0}, \tau_{0}\right)=2^{n / 2} u\left(y_{0}, t_{0}\right) .
$$

Noticing this upper bound, we apply $(2.2)$ to $u$ on $M \times\left[\tau_{0} / 2, \tau_{0}\right]$ and conclude that

$$
\frac{\tau_{0}}{2}\left(\frac{|\nabla u|^{2}}{u^{2}}\right)\left(y, \tau_{0}\right) \leq\left(1+C_{1} \tau_{0}\right)\left(\log \left(\frac{2^{n / 2} u\left(y_{0}, \tau_{0}\right)}{u\left(y, \tau_{0}\right)}\right)+C_{2} \tau_{0}\right) .
$$

Let

$$
g=\log \left(\frac{2^{n / 2} u\left(y_{0}, \tau_{0}\right)}{u\left(y, \tau_{0}\right)}\right)+C_{2} \tau_{0}
$$

The above can be written as

$$
|\nabla \sqrt{g}| \leq \sqrt{\frac{1+C_{1} \tau_{0}}{2 \tau_{0}}}
$$

which implies that

$$
\sup _{B_{\tau_{0}}\left(y_{0}, \sqrt{\tau_{0} /\left(1+C_{1} \tau_{0}\right)}\right)} \sqrt{g}\left(y, \tau_{0}\right) \leq \sqrt{g}\left(y_{0}, \tau_{0}\right)+\frac{1}{\sqrt{2}} .
$$

Rewriting the above in terms of $u$, we have that

$$
u\left(y, \tau_{0}\right) \geq 2^{n / 2} u\left(y_{0}, \tau_{0}\right) \mathrm{e}^{-\left(1 / 2+2 / \sqrt{2} \sqrt{(n / 2) \log 2+C_{2}}\right)}=C_{3} u\left(y_{0}, \tau_{0}\right)
$$

for all $y \in B_{\tau_{0}}\left(y_{0}, \sqrt{\tau_{0} /\left(1+C_{1} \tau_{0}\right)}\right)$. Here we have also used $\tau_{0} \leq 1$. Noticing that

$$
\operatorname{Vol}_{\tau_{0}}\left(B_{\tau_{0}}\left(y_{0}, \sqrt{\frac{\tau_{0}}{1+C_{1} \tau_{0}}}\right)\right) \geq C_{4} \tau_{0}^{n / 2}
$$

for some $C_{4}$ depending on the geometry of $\left(M, g\left(\tau_{0}\right)\right)$. Therefore, we have that

$$
\frac{C_{5}}{\tau_{0}^{n / 2}} \int_{M} u\left(y, \tau_{0}\right) d \mu_{\tau_{0}}(y) \geq u\left(y_{0}, \tau_{0}\right)
$$

for some $C_{5}$ depending on $C_{3}$ and $C_{4}$. By the way we choose $\left(y_{0}, \tau_{0}\right)$, we have that

$$
\tau^{n / 2} u(y, \tau) \leq \tau_{0}^{n / 2} u\left(y_{0}, \tau_{0}\right) \leq C_{5} \int_{M} u\left(y, \tau_{0}\right) d \mu_{\tau_{0}}(y)=C_{5} \int_{M} u(y, \tau) d \mu_{\tau}(y) .
$$


This proves the claim (2.4). Now the estimate (2.3) follows from (2.2), applied to $u$ on $M \times[\tau / 2, \tau]$, and the just proved (2.4), which ensures the needed upper bound for applying the estimate (2.2).

If

$$
u(y, \tau)=\frac{\mathrm{e}^{-f}}{(4 \pi \tau)^{n / 2}}
$$

is the fundamental solution $H\left(y, \tau ; x_{0}, 0\right)$ (expressed in terms of $\tau$ ) to the conjugate heat equation, we have that $\int_{M} u d \mu_{\tau}=1$. Therefore, by (2.3), we have that

$$
\int_{M} \tau|\nabla f|^{2} u h d \mu_{\tau} \leq\left(2+C_{1} \tau\right) \int_{M}\left(\log B+f+\frac{n}{2} \log (4 \pi)+C_{2} \tau\right) u h d \mu_{\tau} .
$$

On the other hand, integrating by parts, we can rewrite

$$
\begin{aligned}
\mathcal{W}_{h}(g, u, \tau)= & \int_{M} \tau|\nabla f|^{2} u h d \mu_{\tau}-2 \tau \int_{M}\langle\nabla f, \nabla h\rangle u d \mu_{\tau}+\tau \int_{M} R u h d \mu_{\tau} \\
& +\int_{M}(f-n) u h d \mu_{\tau} \\
= & \mathrm{I}+\mathrm{II}+\mathrm{III}+\mathrm{IV} .
\end{aligned}
$$

The I term can be estimated by (2.5), whose right hand side contains only one "bad" term $\int_{M} f u h d \mu_{\tau}$ in the sense that it could possibly blow up. The second term

$$
\mathrm{II}=2 \tau \int_{M}\langle\nabla u, \nabla h\rangle d \mu=-2 \tau \int_{M} u \Delta h d \mu_{\tau}
$$

is clearly bounded as $\tau \rightarrow 0$. In fact II $\rightarrow 0$ as $\tau \rightarrow 0$. The same conclusion obviously holds for III. Summarizing above, we reduce the question of bounding from above the quantity $\mathcal{W}_{h}(u, g, \tau)$ to bounding one single term

$$
V=\int_{M} f u h d \mu_{\tau}
$$

from above (as $\tau \rightarrow 0$ ). We shall show later that $\lim _{\tau \rightarrow 0} V \leq 0$. To do this, we need to use the "reduced distance", introduced by Perelman in [1] for the Ricci flow geometry.

Let $x$ be a fixed point in $M$. Let $\ell(y, \tau)$ be the reduced distance in [1] with respect to $(x, 0)$ (more precisely $\tau=0$ ). We collect the relevant properties of $\ell(y, \tau)$ in the following lemma (cf. $[8,10])$. 
Lemma 2.4. Let $\bar{L}(y, \tau)=4 \tau \ell(y, \tau)$.

(i) Assume that there exists a constant $k_{1}$ such that $R_{i j}(g(\tau)) \geq-k_{1} g_{i j}(\tau)$, $\bar{L}(y, \tau)$ is a local Lipschitz function on $M \times[0, T]$;

(ii) Assume that there exist constant $k_{1}$ and $k_{2}$ so that $-k_{1} g_{i j}(\tau) \leq R_{i j}$ $(g(\tau)) \leq k_{2} g_{i j}(\tau)$. Then,

$$
\bar{L}(y, \tau) \leq \mathrm{e}^{2 k_{2} \tau} d_{0}^{2}(x, y)+\frac{4 k_{2} n}{3} \tau^{2}
$$

and

$$
d_{0}^{2}(x, y) \leq \mathrm{e}^{2 k_{1} \tau}\left(\bar{L}(y, \tau)+\frac{4 k_{1} n}{3} \tau^{2}\right)
$$

$$
\left(\frac{\partial}{\partial \tau}-\Delta+R\right)\left(\frac{\exp (-\bar{L}(y, \tau) / 4 \tau)}{(4 \pi \tau)^{n / 2}}\right) \leq 0 .
$$

Proof. The first two claims follow from the definition by straight forward checking. For (iii), it was proved in Section 7 of [1]. By now, there are various sources where a detailed proof can be found; see, for example, $[8,10]$.

As a consequence of (2.6) and (2.7),

$$
\lim _{\tau \rightarrow 0} \frac{\exp (-\bar{L}(y, \tau) / 4 \tau)}{(4 \pi \tau)^{n / 2}}=\delta_{x}(y)
$$

which together with (2.8) implies that $H$, the fundamental solution to the conjugate heat equation, is bounded from below as

$$
H(x, y, \tau) \geq \frac{\exp (-\bar{L}(y, \tau) / 4 \tau)}{(4 \pi \tau)^{n / 2}}
$$

by the heat kernel comparison principle (cf. [11, Proposition 1], noticing the duality between the fundamental solution of the heat equation and the fundamental solution of the conjugate heat equation). Hence,

$$
f(y, \tau) \leq \frac{\bar{L}(y, \tau)}{4 \tau}
$$

This was proved in [1] making use of the inequality $v_{H} \leq 0$. Since we are in the middle of proving $v_{H} \leq 0$, we provide the above alternative of obtaining (2.9). 


\section{Synthesis}

Now we assemble the results in the previous section to prove (1.4). As the first step, we show that $\mathcal{W}_{h}(g, H, \tau)$ is bounded (thanks to the monotonicity (1.3), it is sufficient to bound it from above) as $\tau \rightarrow 0$, where $H(x, y, \tau)$ is the fundamental solution to the conjugate heat equation with $H(x, y, 0)=\delta_{x}(y)$. By the reduction done in the previous section, we only need to show that

$$
V=\int_{M} f H h d \mu_{\tau}
$$

is bounded from above, as $\tau \rightarrow 0$. By (2.9), we have that

$$
\begin{aligned}
& \limsup _{\tau \rightarrow 0} \int_{M} f H h d \mu_{\tau} \\
& \quad \leq \limsup _{\tau \rightarrow 0} \int_{M} \frac{\bar{L}(y, \tau)}{4 \tau} H(x, y, \tau) h(y, \tau) d \mu_{\tau}(y) \\
& \quad \leq \limsup _{\tau \rightarrow 0} \int_{M} \frac{d_{0}^{2}(x, y)}{4 \tau} H(x, y, \tau) h(y, \tau) d \mu_{\tau}(y) \\
& \quad+\lim _{\tau \rightarrow 0} \int_{M}\left(\frac{\mathrm{e}^{k_{2} \tau}-1}{4 \tau} d_{0}^{2}(x, y)+\frac{k_{2} n}{3} \tau\right) H(x, y, \tau) h(y, \tau) d \mu_{\tau}(y) .
\end{aligned}
$$

Here, we have used (2.6) in the last inequality. By Theorem 2.1, some elementary computations give that

$$
\lim _{\tau \rightarrow 0} \int_{M} \frac{d_{0}^{2}(x, y)}{4 \tau} H(x, y, \tau) h(y, \tau) d \mu_{\tau}(y)=\frac{n}{2} h(x, 0) .
$$

Since

$$
\frac{\mathrm{e}^{k_{2} \tau}-1}{4 \tau} d_{0}^{2}(x, y)+\frac{k_{2} n}{3} \tau
$$

is a bounded continuous function even at $\tau=0$, we have that

$$
\lim _{\tau \rightarrow 0} \int_{M}\left(\frac{\mathrm{e}^{k_{2} \tau}-1}{4 \tau} d_{0}^{2}(x, y)+\frac{k_{2} n}{3} \tau\right) H(x, y, \tau) h(y, \tau) d \mu_{\tau}(y)=0 .
$$

This completes our proof of the finiteness of $\lim \sup _{\tau \rightarrow 0} \int_{M} f H h d \mu_{\tau}$. In fact, we have proved that

$$
\limsup _{\tau \rightarrow 0} \int_{M}\left(f-\frac{n}{2}\right) H h d \mu_{\tau} \leq 0
$$


By the just proved finiteness of $\mathcal{W}_{h}(g, H, \tau)$ as $\tau \rightarrow 0$, and the (entropy) monotonicity (1.3), we know that the $\operatorname{limit}_{\lim _{\tau \rightarrow 0}} \mathcal{W}_{h}(g, H, \tau)$ exists. Let

$$
\lim _{\tau \rightarrow 0} \mathcal{W}_{h}(g, H, \tau)=\lim _{\tau \rightarrow 0} \int_{M} v_{H} h d \mu_{\tau}=\alpha
$$

for some finite $\alpha$. Hence $\lim _{\tau \rightarrow 0}\left(\mathcal{W}_{h}(g, H, \tau)-\mathcal{W}_{h}(g, H, \tau / 2)\right)=0$. By (1.3) and the mean-value theorem, we can find $\tau_{k} \rightarrow 0$ such that

$$
\lim _{\tau_{k} \rightarrow 0} \tau_{k}^{2} \int_{M}\left|R_{i j}+\nabla_{i} \nabla_{j} f-\frac{1}{2 \tau_{k}} g_{i j}\right|^{2} H h d \mu_{\tau_{k}}=0
$$

By the Cauchy-Schwartz inequality and the Hölder inequality, we have that

$$
\lim _{\tau_{k} \rightarrow 0} \tau_{k} \int_{M}\left(R+\Delta f-\frac{n}{2 \tau_{k}}\right) H h d \mu_{\tau_{k}}=0 .
$$

This implies that

$$
\lim _{\tau \rightarrow 0} \mathcal{W}_{h}(g, H, \tau)=\lim _{\tau_{k} \rightarrow 0} \int_{M}\left(\tau_{k}\left(\Delta f-|\nabla f|^{2}\right)+f-\frac{n}{2}\right) H h d \mu_{\tau_{k}} .
$$

Again integration by parts shows that

$$
\begin{aligned}
\int_{M} \tau_{k}\left(\Delta f-|\nabla f|^{2}\right) H h d \mu_{\tau_{k}} & =\int_{M} \tau_{k}\langle\nabla H, \nabla h\rangle d \mu_{\tau_{k}} \\
& =-\tau_{k} \int_{M} H \Delta h d \mu_{\tau_{k}} \longrightarrow 0 .
\end{aligned}
$$

Hence, by (3.1),

$$
\lim _{\tau \rightarrow 0} \mathcal{W}_{h}(g, H, \tau)=\lim _{\tau_{k} \rightarrow 0} \int_{M}\left(f-\frac{n}{2}\right) H h d \mu_{\tau_{k}} \leq 0 .
$$

This proves $\alpha \leq 0$, namely (1.4).

The claim that $\alpha=\lim _{\tau \rightarrow 0} \mathcal{W}_{h}(g, H, \tau)=0$ can now be proved by the blow-up argument as in Section 4 of [1]. Assume that $\alpha<0$. One can easily check that this would imply that $\lim _{\tau \rightarrow 0} \mu(g, \tau)<0$. Here $\mu(g, \tau)$ is the invariant defined in Section 4 of [1]. In fact, noticing that $h(y, \tau)>0$ for all $\tau \leq \tau_{*}$ (where $\tau_{*}$ is the one we fixed in the introduction). Therefore, by multiplying $1 / h(x, 0)$ (more precisely $1 / h(x, \cdot)$ at $\tau=0$ ) to the original $h(y, \tau)$, we may assume that $h(x, 0)=\int_{M} H(x, y, \tau) h(y, \tau) d \mu_{\tau}=1$. Let 
$\tilde{u}(y, \tau)=H(x, y, \tau) h(y, \tau)$ and $\tilde{f}=-\log \tilde{u}-n / 2 \log (4 \pi)$. Now, direct computation yields that

$$
\mathcal{W}_{h}(g, H, \tau)=\mathcal{W}(g, \tilde{u}, \tau)+\int_{M}\left(\tau\left(\frac{|\nabla h|^{2}}{h}\right)-h \log h\right) H d \mu_{\tau}
$$

Noticing that the second integration goes to 0 as $\tau \rightarrow 0$, we can deduce that $\mathcal{W}(g, \tilde{u}, \tau)<0$ for sufficient small $\tau$ if $\alpha<0$. This, together with the fact that $\int_{M} \tilde{u} d \mu=1$, implies that $\mu(g, \tau)<0$ for sufficiently small $\tau$. Now Perelman's blow-up argument in Section 4 of [1] gives a contradiction with the sharp logarithmic Sobolev inequality on the Euclidean space [12]. (One can consult, for example, $[4,13]$ for more details of this part.)

Remark 3.1. The method of proof here follows a similar idea used in [4], where the asymptotic limit of the entropy as $\tau \rightarrow \infty$ was computed. Note that we have to use properties of the reduced distance, introduced in Section 7 of [1], in our proof, while the similar, but slightly easier, claim that $\lim _{\tau \rightarrow 0} \mathcal{W}(g, H, \tau)=0$ appears much earlier in Section 4 of [1].

Remark 3.2. Hamilton asked whether or not the LYH-type estimate $v_{u} \leq 0$ still holds for more general positive solution $u$ to the conjugate heat equation, other than the fundamental solution. The proof presented here can be adapted to show that it still holds for finite sum of fundamental solutions. Namely, let $u(y, \tau)=\sum_{i=1}^{k} H\left(y, t ; x_{i}, T\right)$. Then, estimate (1.4), hence $v_{u} \leq 0$, still holds for such $u$.

The proof can be easily modified to give the asymptotic behavior of the entropy defined in [4] for the fundamental solution to the linear heat equation, with respect to a fixed Riemannian metric. Indeed, if we restrict to the class of complete Riemannian manifolds with non-negative Ricci curvature, we have the following estimates.

Proposition 3.3. For any $\delta>0$, there exists $C(\delta)$ such that

$$
\frac{|\nabla H|^{2}}{H}(x, y, \tau) \leq 2 \frac{H(x, y, \tau)}{\tau}\left(C(\delta)+\frac{d^{2}(x, y)}{(4-\delta) \tau}\right)
$$

and

$$
\Delta H(x, y, \tau)+\frac{|\nabla H|^{2}}{H}(x, y, \tau) \leq 2 \frac{H(x, y, \tau)}{\tau}\left(C(\delta)+4 \frac{d^{2}(x, y)}{(4-\delta) \tau}\right)
$$


The previous argument for the Ricci flow case can be transplanted to show that

$$
\tau\left(2 \Delta f-|\nabla f|^{2}\right)+f-n \leq 0,
$$

where

$$
H(y, \tau ; x, 0)=\frac{1}{(4 \pi \tau)^{n / 2}} \mathrm{e}^{-f}
$$

is the fundamental solution to the heat operator $\partial / \partial \tau-\Delta$. This gives a rigorous argument for inequality (1.5) (Theorem 1.2) of [4], for both the compact manifolds and complete manifolds with non-negative Ricci (or Ricci curvature bounded from below). For the full detailed account, please see [8].

\section{Improving $\mathrm{Li}-\mathrm{Yau}-\mathrm{Hamilton}$ estimates via monotonicity formulae}

The proof of (1.4) indicates a close relation between the monotonicity formulae and the differential inequalities of Li-Yau type. The hinge is simply Green's second identity. This was discussed very generally in [5]. Moreover, if we chose $h$ in the introduction to be the fundamental solution to the time dependent heat equation $(\partial / \partial t-\Delta)$ centered at $\left(x_{0}, t_{0}\right)$, we can have a better upper bound on $v_{H}\left(x_{0}, t_{0}\right)$ in terms of the weighted integral which is non-positive. In fact, this follows from the representation formula for the solutions to the non-homogenous conjugate heat equation. More precisely, since $h\left(y, t ; x_{0}, t_{0}\right)$ is the fundamental solution to the heat equation (to make it very clear, $v_{H}$ is defined with respect to $H=H(y, t ; x, T)$, the fundamental solution to the conjugate heat equation centered at $(x, T)$ with $\left.T>t_{0}\right)$, we have that

$$
\lim _{t \rightarrow t_{0}} \int_{M} h\left(y, t ; x_{0}, t_{0}\right) v_{H}(y, t) d \mu_{t}(y)=v_{H}\left(x_{0}, t_{0}\right)
$$

On the other hand, from (1.2), we have that (by Green's second identity)

$$
\frac{d}{d t} \int_{M} h v_{H} d \mu_{t}=2 \tau \int_{M}\left|R_{i j}+f_{i j}-\frac{1}{2 \tau}\right|^{2} H h d \mu_{t}
$$

Therefore,

$$
\lim _{t \rightarrow T} \int_{M} h v_{H} d \mu_{t}-v_{H}\left(x_{0}, t_{0}\right)=\int_{t_{0}}^{T} 2 \tau \int_{M}\left|R_{i j}+f_{i j}-\frac{1}{2 \tau} g_{i j}\right|^{2} H h d \mu_{t} d t .
$$


Using the fact that $\lim _{t \rightarrow T} v_{H}=0$, we have that

$$
v_{H}\left(x_{0}, t_{0}\right)=-2 \int_{t_{0}}^{T}(T-t) \int_{M}\left|R_{i j}+f_{i j}-\frac{1}{2(T-t)}\right|^{2} H h d \mu_{t} d t \leq 0,
$$

which sharpens the estimate $v_{H} \leq 0$ by providing a non-positive upper bound. Noticing also the duality $h\left(y, t ; x_{0}, t_{0}\right)=H\left(x_{0}, t_{0} ; y, t\right)$ for any $t>t_{0}$ (cf. [14]), we can express everything in terms of the fundamental solution to the (backward) conjugate heat equation.

Below we show a few new monotonicity formulae, which expand the list of examples shown in [5] and more importantly improve the earlier established Li-Yau-Hamilton estimates in a similar way as above.

For simplicity, let us just consider the Kähler-Ricci flow case, even though often the discussions are also valid for the Riemannian (Ricci flow) case, after replacing the assumption on the non-negativity of the bisectional curvature by the non-negativity of the curvature operator whenever necessary.

We first let $\left(M, g_{\alpha \bar{\beta}}(x, t)\right)\left(m=\operatorname{dim}_{\mathbb{C}} M\right)$ be a solution to the KählerRicci flow:

$$
\frac{\partial}{\partial t} g_{\alpha \bar{\beta}}=-R_{\alpha \bar{\beta}}
$$

Let $\Upsilon_{\alpha \bar{\beta}}(x, t)$ be a Hermitian symmetric tensor defined on $M \times[0, T]$, which is deformed by the complex Lichnerowicz-Laplacian heat equation (or L-heat equation in short):

$$
\left(\frac{\partial}{\partial t}-\Delta\right) \Upsilon_{\gamma \bar{\delta}}=R_{\beta \bar{\alpha} \gamma \bar{\delta}} \Upsilon_{\alpha \bar{\beta}}-\frac{1}{2}\left(R_{\gamma \bar{p}} k_{p \bar{\delta}}+R_{p \bar{\delta}} \Upsilon_{\gamma \bar{p}}\right)
$$

Let $\operatorname{div}(\Upsilon)_{\alpha}=g^{\gamma \bar{\delta}} \nabla_{\gamma} \Upsilon_{\alpha \bar{\delta}}$ and $\operatorname{div}(\Upsilon)_{\bar{\beta}}=g^{\gamma \bar{\delta}} \nabla_{\bar{\delta}} \Upsilon_{\gamma \bar{\beta}}$. Consider the quantity

$$
\begin{aligned}
Z= & g^{\alpha \bar{\beta}} g^{\gamma \bar{\delta}}\left[\frac{1}{2}\left(\nabla_{\bar{\beta}} \nabla_{\gamma}+\nabla_{\gamma} \nabla_{\bar{\beta}}\right) \Upsilon_{\alpha \bar{\delta}}+R_{\alpha \bar{\delta}} \Upsilon_{\gamma \bar{\beta}}+\left(\nabla_{\gamma} \Upsilon_{\alpha \bar{\delta}} V_{\bar{\beta}}+\nabla_{\bar{\beta}} \Upsilon_{\alpha \bar{\delta}} V_{\gamma}\right)\right. \\
& \left.+\Upsilon_{\alpha \bar{\delta}} V_{\bar{\beta}} V_{\gamma}\right]+\frac{K}{t}
\end{aligned}
$$




$$
\begin{aligned}
= & \frac{1}{2}\left[g^{\alpha \bar{\beta}} \nabla_{\bar{\beta}} \operatorname{div}(\Upsilon)_{\alpha}+g^{\gamma \bar{\delta}} \nabla_{\gamma} \operatorname{div}(\Upsilon)_{\bar{\delta}}\right]+g^{\alpha \bar{\beta}} g^{\gamma \bar{\delta}}\left[R_{\alpha \bar{\delta}} \Upsilon_{\gamma \bar{\beta}}+\nabla_{\gamma} \Upsilon_{\alpha \bar{\delta}} V_{\bar{\beta}}\right. \\
& \left.+\nabla_{\bar{\beta}} \Upsilon_{\alpha \bar{\delta}} V_{\gamma}+\Upsilon_{\alpha \bar{\delta}} V_{\bar{\beta}} V_{\gamma}\right]+\frac{K}{t}
\end{aligned}
$$

where $K$ is the trace of $\Upsilon_{\alpha \bar{\beta}}$ with respect to $g_{\alpha \bar{\beta}}(x, t)$. In [15], the following result, which is the Kähler analog of an earlier result in [16], was showed by the maximum principle.

Theorem 4.1. Let $\Upsilon_{\alpha \bar{\beta}}$ be a Hermitian symmetric tensor satisfying the L-heat equation on $M \times[0, T]$. Suppose $\Upsilon_{\alpha \bar{\beta}}(x, 0) \geq 0$ (and satisfies some growth assumptions in the case $M$ is non-compact). Then, $Z \geq 0$ on $M \times$ $(0, T]$ for any smooth vector field $V$ of type $(1,0)$.

The use of the maximum principle in the proof can be replaced by the integration argument as in the proof of (1.4). For any $T \geq t_{0}>0$, in order to prove that $Z \geq 0$ at $t_{0}$, it suffices to show that when $t=t_{0}, \int_{M} t^{2} Z h d \mu_{t} \geq 0$ for any compact-supported non-negative function $h$. Now we solve the conjugate heat equation $(\partial / \partial \tau-\Delta+R) h(y, \tau)=0$ with $\tau=t_{0}-t$ and $h(y, \tau=$ $0)=h(y)$, the given compact-supported function at $t_{0}$. By the perturbation argument, we may as well as assume that $\Upsilon>0$. Let $Z_{m}(y, t)=\inf _{V} Z(y, t)$. It was shown in [15] that

$$
\left(\frac{\partial}{\partial t}-\Delta\right) Z_{m}=Y_{1}+Y_{2}-2 \frac{Z_{m}}{t}
$$

where

$Y_{1}=\Upsilon_{\bar{p} q}\left(\Delta R_{p \bar{q}}+R_{p \bar{q} \alpha \bar{\beta}} R_{\bar{\alpha} \beta}+\nabla_{\alpha} R_{p \bar{q}} V_{\bar{\alpha}}+\nabla_{\bar{\alpha}} R_{p \bar{q}} V_{\alpha}+R_{p \bar{q} \alpha \bar{\beta}} V_{\bar{\alpha}} V_{\beta}+\frac{R_{p \bar{q}}}{t}\right)$

and

$$
\begin{aligned}
Y_{2} & =\Upsilon_{\gamma \bar{\alpha}}\left[\nabla_{p} V_{\bar{\gamma}}-R_{p \bar{\gamma}}-\frac{1}{t} g_{p \bar{\gamma}}\right]\left[\nabla_{\bar{p}} V_{\alpha}-R_{\alpha \bar{p}}-\frac{1}{t} g_{\bar{p} \alpha}\right]+\Upsilon_{\gamma \bar{\alpha}} \nabla_{\bar{p}} V_{\bar{\gamma}} \nabla_{p} V_{\alpha} \\
& \geq 0
\end{aligned}
$$

Notice that in the above expressions, at every point $(y, t)$, the vector $V(y, t)$ is the vector minimizing $Z_{m}$. This implies the monotonicity

$$
\frac{d}{d t} \int_{M} t^{2} Z_{m} h d \mu_{t}=t^{2} \int_{M}\left(Y_{1}+Y_{2}\right) h d \mu_{t} \geq 0 .
$$


Since $\lim _{t \rightarrow 0} t^{2} Z_{m}=0$, which is certainly the case if $\Upsilon$ is smooth at $t=0$ and can be assumed so in general by shifting $t$ with a $\epsilon>0$, we have that $\left.\int_{M} t^{2} Z_{m} h d \mu\right|_{t=t_{0}} \geq 0$. This proof via the integration by parts implies the following monotonicity formula.

Proposition 4.2. Let $(M, g(t)), \Upsilon$ and $Z$ be as in Theorem 4.1. For any space-time point $\left(x_{0}, t_{0}\right)$ with $0<t_{0} \leq T$, let $\ell(y, \tau)$ be the reduced distance function with respect to $\left(x_{0}, t_{0}\right)$. Then,

$$
\frac{d}{d t} \int_{M} t^{2} Z_{m}\left(\frac{\exp (-\ell)}{(\pi \tau)^{m}}\right) d \mu_{t} \geq t^{2} \int_{M}\left(Y_{1}+Y_{2}\right)\left(\frac{\exp (-\ell)}{(\pi \tau)^{m}}\right) d \mu_{t} \geq 0
$$

In particular,

$$
t_{0}^{2} Z\left(x_{0}, t_{0}\right) \geq \int_{0}^{t_{0}} t^{2}\left(\int_{M}\left(Y_{1}+Y_{2}\right)\left(\frac{\exp (-\ell)}{(\pi \tau)^{m}}\right) d \mu_{t}\right) d t \geq 0
$$

Notice that (4.2) sharpens the original Li-Yau-Hamilton estimate of [15], by encoding the rigidity (such as Hamilton-Cao's characterization on the singularity models), derived out of the equality case in the Li-YauHamilton estimate $Z \geq 0$, into the integral of the right hand side. The result holds for the Riemannian case if one uses computation from [16].

In [3], the author discovered a new matrix Li-Yau-Hamilton inequality for the Kähler-Ricci flow. (We also showed a family of equations which connects this matrix inequality to Perelman's entropy formula.) More precisely, we showed that for any positive solution $u$ to the forward conjugate heat equation $(\partial / \partial t-\Delta-R) u=0$, we have that

$$
\Upsilon_{\alpha \bar{\beta}}:=u\left(\nabla_{\alpha} \nabla_{\bar{\beta}} \log u+R_{\alpha \bar{\beta}}+\frac{1}{t} g_{\alpha \bar{\beta}}\right) \geq 0
$$

under the assumption that $(M, g(t))$ has bounded non-negative bisectional curvature. Using the above argument, we can also obtain a new monotonicity related to (4.3). Indeed, tracing (1.21) of [3] gives that

$$
\left(\frac{\partial}{\partial t}-\Delta\right) Q=R Q-R_{\alpha \bar{\beta}} \Upsilon_{\beta \bar{\alpha}}-\frac{2}{t} Q+\frac{1}{u}\left|\Upsilon_{\alpha \bar{\beta}}\right|^{2}+u\left|\nabla_{\alpha} \nabla_{\beta} \log u\right|^{2}+Y_{3}
$$


where $Q=g_{\alpha \bar{\beta}} \Upsilon_{\alpha \bar{\beta}}$ and

$$
\begin{aligned}
Y_{3}= & u\left(\Delta R+\left|R_{\alpha \bar{\beta}}\right|^{2}+\nabla_{\alpha} R \nabla_{\bar{\alpha}} \log u+\nabla_{\alpha} \log u \nabla_{\bar{\alpha}} R\right. \\
& \left.+R_{\alpha \bar{\beta}} \nabla_{\bar{\alpha}} \log u \nabla_{\beta} \log u+\frac{1}{t} R\right) \\
\geq & 0 .
\end{aligned}
$$

Hence, we have the following monotonicity formula, noticing that

$$
Y_{4}:=R Q-R_{\alpha \bar{\beta}} \Upsilon_{\beta \bar{\alpha}} \geq 0 .
$$

Proposition 4.3. Let $(M, g(t))$ and $\left(x_{0}, t_{0}\right)$ be as in Proposition 4.2. Then,

$$
\begin{aligned}
\frac{d}{d t} \int_{M} t^{2} Q\left(\frac{\exp (-\ell)}{(\pi \tau)^{m}}\right) d \mu_{t} & \geq t^{2} \int_{M}\left(\frac{1}{u}\left|\Upsilon_{\alpha \bar{\beta}}\right|^{2}+u\left|\nabla_{\alpha} \nabla_{\beta} \log u\right|^{2}+Y_{3}+Y_{4}\right) \\
& \times\left(\frac{\exp (-\ell)}{(\pi \tau)^{m}}\right) d \mu_{t} \\
\geq & 0 .
\end{aligned}
$$

In particular,

$t_{0}^{2} Q\left(x_{0}, t_{0}\right) \geq \int_{0}^{t_{0}} t^{2} \int_{M}\left(\frac{1}{u}\left|\Upsilon_{\alpha \bar{\beta}}\right|^{2}+u\left|\nabla_{\alpha} \nabla_{\beta} \log u\right|^{2}+Y_{3}+Y_{4}\right)\left(\frac{\exp (-\ell)}{(\pi \tau)^{m}}\right)$.

Again the advantage of the above monotonicity formula is that it encodes the consequence on equality case (which is that $(M, g(t))$ is a gradient expanding soliton) into the right hand side integral.

Without Ricci flow, we can apply the similar argument to prove LiYau's inequality and obtain a monotonicity formula. More precisely, let $(M, g)\left(n=\operatorname{dim}_{\mathbb{R}} M\right)$ be a complete Riemannian manifold with non-negative Ricci curvature. Let $u(x, t)$ be a positive solution to the heat equation on $M \times[0, T] . \mathrm{Li}$ and Yau proved that

$$
\Delta \log u+\frac{n}{2 t} \geq 0 .
$$

Another way of proving the above Li-Yau's inequality is through the above integration by parts argument and the differential equation

$$
\left(\frac{\partial}{\partial t}-\Delta\right) Q=\frac{2}{u}\left|\Upsilon_{i j}\right|^{2}-\frac{2}{t} Q+\frac{2}{u} R_{i j} \nabla_{i} u \nabla_{j} u
$$


where

$$
\Upsilon_{i j}=\nabla_{i} \nabla_{j} u+\frac{u}{2 t} g_{i j}-\frac{u_{i} u_{j}}{u}
$$

and $Q=g_{i j} \Upsilon_{i j}=u(\Delta \log u+n / 2 t)$. This together with Cheeger-Yau's theorem [17] on lower bound of the heat kernel gives the following monotonicity formula, which also give characterization on the manifold if the equality holds somewhere for some positive $u$.

Proposition 4.4. Let $(M, g)$ be a complete Riemannian manifold with non-negative Ricci curvature. Let $\left(x_{0}, t_{0}\right)$ be a space-time point with $t_{0}>0$. Let $\tau=t_{0}-t$. Then,

$$
\begin{aligned}
& \frac{d}{d t}\left(\int_{M} t^{2} Q(y, t) \hat{H}\left(x_{0}, y, \tau\right) d \mu(x)\right) \geq 2 t^{2} \int_{M}\left(\left|\nabla_{i} \nabla_{j} \log u+\frac{1}{2 t} g_{i j}\right|^{2}\right. \\
& \left.\quad+R_{i j} \nabla_{i} \log u \nabla_{j} \log u\right) u \hat{H} d \mu \geq 0
\end{aligned}
$$

where

$$
\hat{H}\left(x_{0}, y, \tau\right)=\frac{1}{(4 \pi \tau)^{n / 2}} \exp \left(-\frac{d^{2}\left(x_{0}, y\right)}{4 \tau}\right)
$$

with $d\left(x_{0}, y\right)$ being the distance function between $x_{0}$ and $y$. In particular, we have that

$$
\begin{aligned}
\left(u \Delta \log u+\frac{n}{2 t} u\right)\left(x_{0}, t_{0}\right) \geq & \frac{2}{t_{0}^{2}} \int_{0}^{t_{0}} t^{2} \int_{M}\left(\left|\nabla_{i} \nabla_{j} \log u+\frac{1}{2 t} g_{i j}\right|^{2}\right. \\
& \left.+R_{i j} \nabla_{i} \log u \nabla_{j} \log u\right) u \hat{H}
\end{aligned}
$$

It is clear that (4.7) improves the estimate of Li-Yau slightly by providing the lower estimate, from which one can see easily that the equality (for Li-Yau's estimate) holding somewhere implies that $M=\mathbb{R}^{n}$ (this was first observed in [4], with the help of an entropy formula). The expression in the right hand side of (4.6) also appears in the linear entropy formula of [4].

One can write down similar improving results for the Li-Yau-type estimate proved in [4], which is a linear analog of Perelman's estimate $v_{H} \leq 0$, and the one in [18], which is a linear version of Theorem 4.1. For example, when $M$ is a complete Riemannian manifold with the non-negative 
Ricci curvature, if

$$
u=H(x, y, t)=\frac{\mathrm{e}^{-f}}{(4 \pi t)^{n / 2}},
$$

the fundamental solution to the heat equation centered at $x$ at $t=0$, letting $W=t(2 \Delta f-|\nabla f|)+f-n$, we have that $W \leq 0$. If $\hat{H}$ is the "pseudobackward heat kernel" defined as in Proposition 4.4, we have that

$$
\begin{aligned}
& \frac{d}{d t} \int_{M}(-W) u \hat{H}\left(x_{0}, y, \tau\right) d \mu(y) \\
& \quad=2 t \int_{M}\left(\left|\nabla_{i} \nabla_{j} f-\frac{1}{2 t} g_{i j}\right|^{2}+R_{i j} \nabla_{i} f \nabla_{j} f\right) u \hat{H}\left(x_{0}, y, \tau\right) d \mu(y) \geq 0
\end{aligned}
$$

and

$$
(-W u)\left(x_{0}, t_{0}\right) \geq 2 \int_{0}^{t_{0}} t \int_{M}\left(\left|\nabla_{i} \nabla_{j} f-\frac{1}{2 t} g_{i j}\right|^{2}+R_{i j} \nabla_{i} f \nabla_{j} f\right) u \hat{H} .
$$

If we assume further that $M$ is a complete Kähler manifold with non-negative bisectional curvature and $u(y, t)$ is a strictly plurisubharmonic solution to the heat equation with $w=u_{t}$, then

$$
\frac{d}{d t} \int_{M} t^{2} Z_{m}^{w} \hat{H}\left(x_{0}, y, t\right) d \mu(y)=t^{2} \int_{M} Y_{5} \hat{H}\left(x_{0}, y, t\right) d \mu(y) \geq 0
$$

where

$$
Z_{m}^{w}(y, t)=\inf _{V \in T^{1,0} M}\left(w_{t}+\nabla_{\alpha} w V_{\bar{\alpha}}+\nabla_{\bar{\alpha}} w V_{\alpha}+u_{\alpha \bar{\beta}} V_{\bar{\alpha}} V_{\beta}+\frac{w}{t}\right)
$$

and

$$
\begin{aligned}
Y_{5} & =u_{\gamma \bar{\alpha}}\left[\nabla_{p} V_{\bar{\gamma}}-\frac{1}{t} g_{p \bar{\gamma}}\right]\left[\nabla_{\bar{p}} V_{\alpha}-\frac{1}{t} g_{\bar{p} \alpha}\right]+u_{\gamma \bar{\alpha}} \nabla_{\bar{p}} V_{\bar{\gamma}} \nabla_{p} V_{\alpha}+R_{\alpha \bar{\beta} s \bar{t}} u_{\bar{s} t} V_{\beta} V_{\bar{\alpha}} \\
& \geq 0
\end{aligned}
$$

with $V$ being the minimizing vector in the definition of $Z_{m}^{w}$. In particular,

$$
\left(\frac{\partial^{2}}{\partial(\log t)^{2}} u(y, t)\right)\left(x_{0}, t_{0}\right) \geq \int_{0}^{t_{0}} t^{2} \int_{M} Y_{5} \hat{H}\left(x_{0}, y, t\right) d \mu(y) d t .
$$

This sharpens the logarithmic convexity of $u(y, t)$ proved in [17]. 
Finally, we should remark that in all the discussions above, one can replace the pseudo-backward heat kernel

$$
\hat{H}\left(y, t ; x_{0}, t_{0}\right)=\frac{\exp \left(-d^{2}\left(x_{0}, y\right) / 4\left(t_{0}-t\right)\right)}{\left(4 \pi\left(t_{0}-t\right)\right)^{n / 2}}
$$

(or $\exp (-\ell(y, \tau)) /(4 \pi \tau)^{n / 2}$, centered at $\left(x_{0}, t_{0}\right)$ in the case of Ricci flow), which we wrote before as $\hat{H}\left(y, x_{0}, \tau\right)$ by abusing the notation, by the fundamental solution to the backward heat equation (even by constant 1 in the case of compact manifolds). Also, it still remains interesting on how to make effective use of these improved estimates, besides the rigidity results out of the inequality being equality somewhere. There is also a small point that should not be glossed over. When the manifold is complete non-compact, one has to justify the validity of Green's second identity (for example, in Proposition 4.4 , we need to justify that $\left.\int_{M}(\hat{H} \Delta Q-Q \Delta \hat{H}) d \mu=0\right)$. This can be done when $t_{0}$ is sufficiently small, together with integral estimates on the Li-Yau-Hamilton quantity (cf. [8]). The local monotonicity formula that shall be discussed in the next section provides another way to avoid possible technical complications caused by the non-compactness.

\section{Local monotonicity formulae}

In [5], a very general scheme on localizing the monotonicity formulae is developed. It is for any family of metrics evolved by the equation $\partial / \partial t g_{i j}=$ $-2 \kappa_{i j}$. The localization is through the so-called "heat ball". More precisely, for a smooth positive space-time function $v$, which often is the fundamental solution to the backward conjugate heat equation or the pseudo-backward heat kernel

$$
\hat{H}\left(x_{0}, y, \tau\right)=\frac{\mathrm{e}^{-r^{2}\left(x_{0}, y\right) / 4 \tau}}{(4 \pi \tau)^{n / 2}}
$$

(or $\mathrm{e}^{-\ell(y, \tau)} /(4 \pi \tau)^{n / 2}$ in the case of Ricci flow), with $\tau=t_{0}-t$, one defines the heat ball by $E_{r}=\left\{(y, t) \mid v \geq r^{-n} ; t<t_{0}\right\}$. For all interesting cases, we can check that $E_{r}$ is compact for small $r$ (cf. [5]). Let $\psi_{r}=\log v+n \log r$. For any Li-Yau-Hamilton quantity $\mathcal{Q}$, we define the local quantity:

$$
P(r):=\int_{E_{r}}\left(\left|\nabla \psi_{r}\right|^{2}+\psi_{r}\left(\operatorname{tr}_{g} \kappa\right)\right) \mathcal{Q} d \mu_{t} d t .
$$


The finiteness of the integral can be verified via the localization of Lemma 2.2, a local gradient estimate. The general form of the theorem, which is proved in Theorem 1 of [5], reads as the following.

Theorem 5.1. Let

$$
I(r)=\frac{P(r)}{r^{n}}
$$

Then,

$$
\begin{aligned}
I\left(r_{2}\right)-I\left(r_{1}\right)= & -\int_{r_{1}}^{r_{2}} \frac{n}{r^{n+1}} \int_{E_{r}}\left[\left(\left(\frac{\partial}{\partial t}+\Delta-\operatorname{tr}_{g} \kappa\right) v\right) \frac{\mathcal{Q}}{v}\right. \\
& \left.+\psi_{r}\left(\frac{\partial}{\partial t}-\Delta\right) \mathcal{Q}\right] d \mu_{t} d t d r .
\end{aligned}
$$

It gives the monotonicity of $I(r)$ in the cases that $\mathcal{Q} \geq 0$, which is ensured by the $\mathrm{Li}-\mathrm{Yau}-\mathrm{Hamilton}$ estimates in the case we shall consider, and both $\left(\partial / \partial t+\Delta-\operatorname{tr}_{g} \kappa\right) v$ and $(\partial / \partial t-\Delta) \mathcal{Q}$ are non-negative. The nonnegativity of $\left(\partial / \partial t+\Delta-\operatorname{tr}_{g} \kappa\right) v$ comes for free if we chose $v$ to be the pseudo-backward heat kernel. The non-negativity of $(\partial / \partial t-\Delta) \mathcal{Q}$ follows from the computation, which we may call as in [3] the pre-Li-Yau-Hamilton equation, during the proof of the corresponding $\mathrm{Li}-\mathrm{Yau}-\mathrm{Hamilton}$ estimate. Below, we illustrate examples corresponding to the monotonicity formulae derived in the previous section. These new ones expand the list of examples given in Section 4 of [5].

For the case of Ricci/Kähler-Ricci flow, for a fixed $\left(x_{0}, t_{0}\right)$, let

$$
v=\frac{\mathrm{e}^{-\ell(y, \tau)}}{(4 \pi \tau)^{n / 2}}
$$

the pseudo-backward heat kernel, where $\ell$ is the reduced distance centered at $\left(x_{0}, t_{0}\right)$.

Example 5.2. Let $Z_{m}, Y_{1}$ and $Y_{2}$ be as in Proposition 4.2. Let $\mathcal{Q}=t^{2} Z_{m}$. Then,

$$
\frac{d}{d r} I(r) \leq-\frac{n}{r^{n+1}} \int_{E_{r}}\left[t^{2} \psi_{r}\left(Y_{1}+Y_{2}\right)\right] d \mu_{t} d t \leq 0
$$

and

$$
\mathcal{Q}\left(x_{0}, t_{0}\right) \geq I(\bar{r})+\int_{0}^{\bar{r}} \frac{n}{r^{n+1}} \int_{E_{r}}\left[t^{2} \psi_{r}\left(Y_{1}+Y_{2}\right)\right] d \mu_{t} d t d r
$$


Example 5.3. Let $u, \mathcal{Q}=t^{2} Q, \Upsilon_{\alpha \bar{\beta}}, Y_{3}$ and $Y_{4}$ be as in Proposition 4.3.

Then,

$$
\frac{d}{d r} I(r) \leq-\frac{n}{r^{n+1}} \int_{E_{r}} t^{2} \psi_{r}\left(\frac{1}{u}\left|\Upsilon_{\alpha \bar{\beta}}\right|^{2}+u\left|\nabla_{\alpha} \nabla_{\beta} \log u\right|^{2}+Y_{3}+Y_{4}\right) \leq 0
$$

and

$$
\mathcal{Q}\left(x_{0}, t_{0}\right) \geq I(\bar{r})+\int_{0}^{\bar{r}} \frac{n}{r^{n+1}} \int_{E_{r}} t^{2} \psi_{r}\left(\frac{1}{u}\left|\Upsilon_{\alpha \bar{\beta}}\right|^{2}+u\left|\nabla_{\alpha} \nabla_{\beta} \log u\right|^{2}+Y_{3}+Y_{4}\right) .
$$

For the fixed metric case, we may choose either $v=H\left(x_{0}, y, \tau\right)$, the backward heat kernel or

$$
v=\hat{H}\left(x_{0}, y, \tau\right)=\frac{\mathrm{e}^{-d^{2}\left(x_{0}, y\right) / 4 \tau}}{(4 \pi \tau)^{n / 2}},
$$

the pseudo-backward heat kernel.

Example 5.4. Let $u$ and $Q$ be as in Proposition 4.4. Let $\mathcal{Q}=t^{2} Q$ and $f=\log u$. Then,

$$
\frac{d}{d r} I(r) \leq-\frac{2 n}{r^{n+1}} \int_{E_{r}} t^{2} u \psi_{r}\left(\left|\nabla_{i} \nabla_{j} f+\frac{1}{2 t} g_{i j}\right|^{2}+R_{i j} \nabla_{i} f \nabla_{j} f\right) d \mu d t \leq 0
$$

and

$$
\mathcal{Q}\left(x_{0}, t_{0}\right) \geq I(\bar{r})+\int_{0}^{\bar{r}} \frac{2 n}{r^{n+1}} \int_{E_{r}} t^{2} u \psi_{r}\left(\left|\nabla_{i} \nabla_{j} f+\frac{1}{2 t} g_{i j}\right|^{2}+R_{i j} \nabla_{i} f \nabla_{j} f\right) .
$$

Example 5.5. Let $u=\mathrm{e}^{-f} /(4 \pi t)^{n / 2}$ be the fundamental solution to the (regular) heat equation. Let $W=t\left(2 \Delta f-|\nabla f|^{2}\right)+f-n$ and $\mathcal{Q}=-u W$. Then,

$$
\frac{d}{d r} I(r) \leq-\frac{2 n}{r^{n+1}} \int_{E_{r}} t u \psi_{r}\left(\left|\nabla_{i} \nabla_{j} f-\frac{1}{2 t} g_{i j}\right|^{2}+R_{i j} \nabla_{i} f \nabla_{j} f\right) d \mu d t \leq 0
$$

and

$$
\mathcal{Q}\left(x_{0}, t_{0}\right) \geq I(\bar{r})+\int_{0}^{\bar{r}} \frac{2 n}{r^{n+1}} \int_{E_{r}} t u \psi_{r}\left(\left|\nabla_{i} \nabla_{j} f-\frac{1}{2 t} g_{i j}\right|^{2}+R_{i j} \nabla_{i} f \nabla_{j} f\right) .
$$

Note that this provides another localization of entropy, other than the one in [3] (see also [8]). 
Example 5.6. Let $M$ be a complete Kähler manifold with non-negative bisectional curvature. Let $u, Z_{m}^{w}$ and $Y_{5}$ be as in the last case considered in Section 4. Let $\mathcal{Q}=t^{2} Z_{m}^{w}$. Then,

$$
\frac{d}{d r} I(r) \leq-\frac{n}{r^{n+1}} \int_{E_{r}} t^{2} Y_{5} \psi_{r} d \mu d t
$$

and

$$
\left(\frac{\partial^{2}}{\partial(\log t)^{2}} u(x, t)\right)\left(x_{0}, t_{0}\right) \geq I(\bar{r})+\int_{0}^{\bar{r}} \frac{n}{r^{n+1}} \int_{E_{r}} t^{2} Y_{5} \psi_{r} d \mu d t d r
$$

\section{Acknowledgment}

We would like to thank Ben Chow and Peng Lu for continuously pressing us on a understandable proof of (1.4). We started to seriously work on it after the visit to Klaus Ecker in August and a stimulating discussion with him. We would like to thank him, Dan Knopf and Peter Topping for discussions on a related issue. We are also grateful to Ben Chow for correcting an error in deriving Lemma 2.2 in the earlier version, and Richard Hamilton for his interests and support to this work. The author was supported in part by NSF Grants and an Alfred P. Sloan Fellowship, USA.

\section{References}

[1] G. Perelman, The entropy formula for the Ricci flow and its geometric applications, arXiv: math.DG/0211159.

[2] P. Li and S.-T. Yau, On the parabolic kernel of the Schrödinger operator, Acta Math. 156 (1986), nos. 3, 4, 153-201.

[3] L. Ni, A new matrix Li-Yau-Hamilton inequality for Kähler-Ricci flow, J. Diff. Geom., to appear.

[4] L. Ni, The entropy formula for linear heat equation, J. Geom. Anal. 14 (2004), 87-100; Addenda, 14 (2004), 369-374.

[5] K. Ecker, D. Knopf, L. Ni and P. Topping, Local monotonicity and mean value formulas for evolving Riemanniann manifolds, submitted.

[6] N. Garofalo and E. Lanconelli, Asymptotic behavior of fundamental solutions and potential theory of parabolic operators with variable coefficients, Math. Ann. 283 (1989), no. 2, 211-239. 
[7] R. Schoen and S.-T. Yau, Lectures on differential geometry, Conference Proceedings and Lecture Notes in Geometry and Topology, I, International Press, Cambridge, MA, 1994, pp. v+235.

[8] B. Chow, P. Lu and L. Ni, Hamilton's Ricci Flow, vol. 1 and 2, Science Press/AMS Press, to appear.

[9] R. Hamilton, A matrix Harnack estimate for the heat equation, Comm. Anal. Geom. 1 (1993), no. 1, 113-126.

[10] R. Ye, Notes on reduced volume and asymptotic Ricci solitons of $\kappa$-solutions, http://www.math.lsa.umich.edu/research/ricciflow/ perelman.html.

[11] S.-Y. Cheng, P. Li and S.-T. Yau, Heat equations on minimal submanifolds and their applications, Amer. J. Math. 103 (1984), no. 5, 1033-1065.

[12] L. Gross, Logarithmic Sobolev inequalities, Amer. J. Math. 97 (1975), no. 4, 1061-1083.

[13] N. Sesum, G. Tian and X. Wang, Notes on Perelman's paper.

[14] A. Friedman, Partial Differential Equations of Parabolic Type, Robert E. Krieger Publishing Company, Malabar, Florida, 1983.

[15] L. Ni and L.-F. Tam, Plurisubharmonic functions and the Kähler-Ricci flow, Amer. J. Math. 125 (2003), 623-654 .

[16] B. Chow and R. Hamilton, Constrained and linear Harnack inqualities for parabolic equations, Invent. Math. 129 (1997), 213-238.

[17] J. Cheeger and S.-T. Yau, A lower bound for the heat kernel, Comm. Pure Appl. Math. 34 (1981), no. 4, 465-480.

[18] L. Ni, A monotonicity formula on complete Kähler manifold with nonnegative bisectional curvature, J. Amer. Math. Soc. 17 (2004), 909-946.

Department of Mathematics

University of California at San Diego

LA JOLLA

CA 92093

USA

E-mail address: lni@math.ucsd.edu

Received September 9, 2005 
